\title{
More than one way....
}

Three recent papers that have used combined genomics approaches describe important progress towards the ultimate goal of understanding all genetic changes occurring within specific tumour types.

The Cancer Genome Atlas (TCGA) aims to discover and catalogue major cancer-causing genomic alterations. Glioblastoma is one of the first tumour types to be tackled by this consortium: 206 patient samples were analysed for DNA methylation status, copy-number aberrations and gene expression. This integrative approach identified previously known causative changes as well as novel genetic alterations, such as homozygous deletion of $\underline{N F 1}$ and PARK2 and amplification of AKT3.

Of these samples, 91 were selected for sequencing to look for somatic mutations in 601 genes of interest. Altogether, 223 genes were found to have non-silent mutations, and 79 of these genes had more than one mutation. Interestingly, the mutation rate differed markedly between untreated and treated patients, with 7 of the treated patients having a hypermutated phenotype. Based on their findings, the authors identified a potential link between loss of a DNA-repair pathway that generates sensitivity to treatment with an alkylating agent, hypermutation and disruption of the DNA mismatch repair (MMR) pathway. This connection raises the possibility that resistance to the drug in question might arise through selection for MMR pathway mutations.

Another consortium - including Bert Vogelstein, Victor Velculescu and Kenneth Kinzler - performed sequencing and gene expression analyses on glioblastoma and pancreatic cancer samples. After sequencing 20,661 protein-coding genes in 22 human glioblastoma samples, they noted that one sample from a treated patient had 17 -fold more alterations than the untreated patients and a dramatically different mutation spectrum. Analysis of the 21 untreated samples revealed a mean of 47 mutations per tumour - less than previously established for colorectal or breast tumours, suggesting that glioblastoma arises after fewer cell divisions than these epithelial cancers.

In the same study, combined assessment of sequencing, copy number and gene expression data identified the mutations most likely to drive cancer development. These included changes to genes involved in the pathways highlighted by the TCGA study, and novel changes in genes that regulate neuron-specific functions. Analysis of individual gene data showed that isocitrate dehydrogenase 1 (IDH1), which is involved in the regulation of oxidative damage through production of NADPH, was mutated in $12 \%$ of all samples analysed. Interestingly, this gene seems to be more often mutated in younger patients, and this is correlated with an improved prognosis.

A similar series of analyses in 24 advanced pancreatic adenocarcinoma samples showed that 1,327 of the 20,661 genes sequenced had at least one mutation, with a mean of 48 per tumour. Interestingly, large genomic deletions are commonly found in pancreatic tumours, with
CDKN2A (encoding INK4A and ARF), TP53 and SMAD4 being the most likely tumour suppressors to be selected against. Analysis of driver mutations identified a number of genes known to be associated with pancreatic cancer and many that are not. Differential gene expression analyses also showed that 541 genes were overexpressed, 54 of which encode secreted or cell-surface proteins, making them potential diagnostic and therapeutic targets. The authors developed an algorithm to link mutations to cellular pathways and found that, although there are 12 core signalling pathways and processes that are disrupted in pancreatic cancer, the genetic changes responsible for them vary widely between individual tumours. Therefore, therapies that target a pathway rather than a gene are likely to be more effective.

All three papers clearly show that unbiased systematic approaches can lead to a more comprehensive understanding of the changes that occur during tumour development and treatment.

Nicola McCarthy, Chief Editor, Nature Reviews Cancer

\section{ORIGINAL RESEARCH PAPERS}

The Cancer Genome Atlas Research Network. Comprehensive genomic characterization defines human glioblastoma genes and core pathways. Nature 4 Sep 2008 (doi:10.1038/ nature07385) | Williams Parsons, D. et al. An integrated genomic analysis of human glioblastoma multiforme. Science 4 Sep 2008 (doi:10.1126/science.1164382)| Jones, S. et al. Core signalling pathways in human pancreatic cancers revealed by global genomic analyses. Science 4 Sep 2008 (doi:10.1126/science.1164368) WEB SITE

The Cancer Genome Atlas:

http://cancergenome.nih.gov 